\title{
Linear negative magnetoresistance in two-dimensional Lorentz gases
}

\author{
J. Schluck, M. Hund, T. Heckenthaler, and T. Heinzel ${ }^{*}$ \\ Condensed Matter Physics Laboratory, Heinrich-Heine-Universität, D-40204 Düsseldorf, Germany \\ N. H. Siboni and J. Horbach \\ Institut für Theoretische Physik II, Heinrich-Heine-Universität, D-40204 Düsseldorf, Germany \\ K. Pierz and H. W. Schumacher \\ Physikalisch-Technische Bundesanstalt, Bundesallee 100, D-38116 Braunschweig, Germany \\ D. Kazazis, ${ }^{\dagger}$ U. Gennser, and D. Mailly \\ C2N-CNRS and UPSud, Route de Nozay, 91460 Marcoussis, France
}

(Received 15 December 2017; revised manuscript received 21 February 2018; published 5 March 2018)

\begin{abstract}
Two-dimensional Lorentz gases formed by obstacles in the shape of circles, squares, and retroreflectors are reported to show a pronounced linear negative magnetoresistance at small magnetic fields. For circular obstacles at low number densities, our results agree with the predictions of a model based on classical retroreflection. In extension to the existing theoretical models, we find that the normalized magnetoresistance slope depends on the obstacle shape and increases as the number density of the obstacles is increased. The peaks are furthermore suppressed by in-plane magnetic fields as well as by elevated temperatures. These results suggest that classical retroreflection can form a significant contribution to the magnetoresistivity of two-dimensional Lorentz gases, while contributions from weak localization cannot be excluded, in particular for large obstacle densities.
\end{abstract}

DOI: 10.1103/PhysRevB.97.115301

\section{INTRODUCTION}

The magnetoresistivity of two-dimensional electron gases defined in semiconductor heterostructures can show pronounced deviations from the behavior expected within the Drude-Boltzmann model, i.e., a constant longitudinal and a linear Hall magnetoresistivity. The deviations can be traced back to various origins. For example, the quantization of the cyclotron motion in a perpendicular magnetic field $B_{\perp}$ lies at the heart of the quantum Hall effect and of the Shubnikovde Haas oscillations in the longitudinal resistivity $\rho_{x x}\left(B_{\perp}\right)$ [1], while weak localization increases $\rho_{x x}\left(B_{\perp}\right)$ at $B_{\perp}=0$, which decays in a characteristic, nonlinear way as $B_{\perp}$ is increased [2]. Weak localization also occurs in disordered arrays of classical scatterers as a consequence of an interplay between classical chaos and interference of the electron waves $[3,4]$. Interaction effects typically generate a relatively broad negative, approximately parabolic correction to $\rho_{x x}\left(B_{\perp}\right)$ [5]. Within a hydrodynamic model that includes a $B$-dependent viscosity of the electron liquid, interactions have been used to explain the giant negative magnetoresistance (GNMR) [6], observed in two-dimensional electron gases with high electron mobilities [7-11]. One further possible origin of a negative magnetoresistance are memory effects. They are caused by time-independent components in the scattering potential which

\footnotetext{
*thomas.heinzel@hhu.de

${ }^{\dagger}$ Present address: Laboratory for Micro-PLXINSERT-, and Nanotechnology, Paul Scherrer Institute, 5232 Villigen-PSI, Switzerland.
}

do not randomize the electron momentum in the sense of the relaxation time approximation and thus generate deviations from the Boltzmann expressions for the magnetoresistivities [12]. A suitable model system for studying memory effects, which have a classical character, is an electron gas exposed to a static, Poisson-distributed array of identical obstacles. It was first proposed by Lorentz [13] and is therefore known as Lorentz gas. Two-dimensional Lorentz gases (2DLGs) are of particular interest since a perpendicular magnetic field has profound effects on their transport properties. Several theoretical studies on the magnetotransport properties of 2DLGs have been reported, which has resulted in the identification of various memory effects [14-19]. A prominent example are electrons that do not contribute to the longitudinal transport since they move forever in an undisturbed cyclotron orbit. For circular obstacles acting as scatterers at low number densities $n_{s}$, it has been shown that this memory effect causes an exponential decrease of $\rho_{x x}$ as $B_{\perp}$ increases [14,15]. Such analytic expressions originate from kinematic models which require a dimensionless obstacle number density $n^{\star}=\frac{1}{4} n_{s} d_{s}^{2}$ to be small compared to 1 . Here, $d_{s}$ denotes the characteristic size of the scatterers. Extensions to larger number densities have been carried out numerically for circular obstacles, using molecular dynamics simulations. They have revealed that the conductivity as a function of $n^{\star}$ and $B_{\perp}$ forms a delocalized, conductive phase, which is sandwiched between two localized phases at low and at high obstacle densities [20-22].

Of particular relevance to this study are the works of Dmitriev et al. [18] and Cheianov et al. [19] on retroreflection in dilute 2DLGs with circular obstacles. This type of memory 
(a)

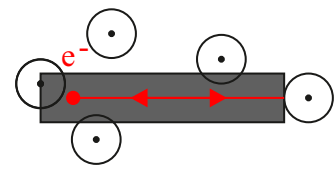

(b)

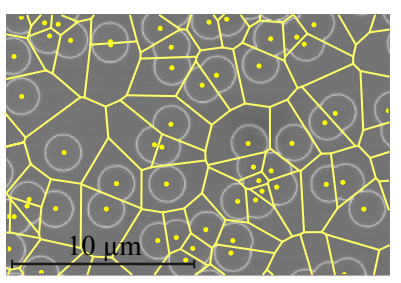

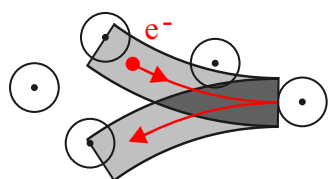

(c)

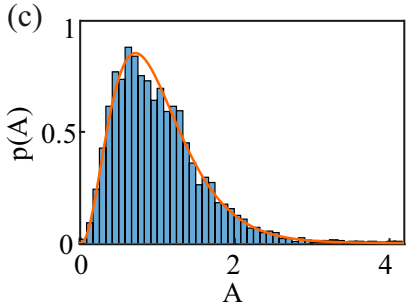

FIG. 1. (a) Illustration of the memory term caused by retroreflection at $B_{\perp}=0$ (left) and $B_{\perp}>0$ (right). The electron moves through the obstacles (circles) which define the ballistic corridors before and after backscattering (light gray, right), with their overlap area marked in dark gray. (b) Scanning electron micrograph of the 2DLG with circular obstacles of radius $d_{s}=2 \mu \mathrm{m}$ and $n_{s}=1.95 \times 10^{11} \mathrm{~m}^{-2}$ $\left(n^{\star}=0.195\right)$. The obstacle centers and the corresponding Voronoi tessellation are shown as an overlay. (c) Histogram of the normalized Voronoi cell areas $A$ for our arrays with number density $n_{s}=$ $1.95 \times 10^{11} \mathrm{~m}^{-2}$, and the fit (red curve) to the probability density function $p(A)$ as described in the text.

effect can be understood in terms of an electron that gets scattered at an obstacle by an angle close to $\pi$ after having traveled a certain distance $L$ ballistically. This distance is related to a two-dimensional corridor of area $L \times d_{s}$ which is free of obstacle centers [see Fig. 1(a) for an illustration]. After the electron has undergone backscattering, it probes the same corridor again which is still free of obstacles, due to the static character of the scattering potential. This memory term increases the resistance since any scattering event at time-dependent potentials in this corridor would give the electron a momentum component in forward direction. The overlap between the corridors related to the incoming and the backscattered electron trajectory [see Fig. 1(a)] equals the area probed twice, and is thus a measure of this memory term. As $B_{\perp}$ is increased, this overlap decreases due to the Lorentz deflection. Consequently, $B_{\perp}$ suppresses this memory term and causes the resistance to decrease. Remarkably, this negative magnetoresistivity has been predicted to be linear [18]. Experiments by Gusev et al. [23] are in agreement with this prediction. Magnetotransport in 2DLGs formed by arrays of circular obstacles has been studied experimentally by Yevtushenko et al. [3], also at relatively low $n^{\star}$. The authors observe a negative magnetoresistance which they explain by ballistic weak localization. This takes into account correlations in the electronic motion as well as the classical character of the obstacles. Due to their much larger size than the Fermi wavelength, they do not split the wave functions in time-reversed backscattering paths as assumed within the conventional weak localization description. However, it has been argued later on that this phenomenology can also be explained by classical retroreflection [18]. Thus, the relevance of these two mechanisms, namely, classical retroreflection and quantum mechanical ballistic weak localization, for the observed negative magnetoresistance of two-dimensional electron gases exposed to random arrays of classical obstacles is still an open question.

Here, we report magnetotransport measurements on 2DLGs composed of classical scatterers. We find an approximately linear negative magnetoresistivity close to $B_{\perp}=0$. The dependencies of the peaks on the parameters shape and density of the obstacles, temperature as well as on in-plane magnetic field is reported. Our data suggest, in brief, that classical retroreflection does contribute significantly to the measured peaks while a contribution of ballistic weak localization cannot be excluded.

The paper is organized as follows. In Sec. II, we describe the sample preparation and the measurement setup. The experimental results are presented and interpreted in Sec. III. We end with a summary and conclusion in Sec. IV.

\section{SAMPLE PREPARATION AND EXPERIMENTAL SETUP}

A $\mathrm{GaAs} / \mathrm{Al}_{0.3} \mathrm{Ga}_{0.7} \mathrm{As}$ heterostructure with a twodimensional electron gas (2DEG) $150 \mathrm{~nm}$ below the surface is used. The pristine 2DEG has a density of $2.5 \times 10^{15} \mathrm{~m}^{-2}$ and an electron mobility of $340 \mathrm{~m}^{2} / \mathrm{Vs}$, corresponding to a mean-free path of $\ell=31 \mu \mathrm{m}$ at liquid helium temperatures. The quantum scattering time as determined from the envelope of the Shubnikov-de Haas oscillations [24] amounts to $\tau_{q}=2.4 \mathrm{ps}$ which gives a quantum scattering length of $\ell_{q}=530 \mathrm{~nm}$. The Lorentz array is prepared by generating two sets of random numbers which define the $x$ and $y$ coordinates of the obstacle centers. The correct statistical properties of the arrays are checked by a Voronoi tessellation of the array, where each obstacle center defines a cell containing all points closer to this center than to any other [see Fig. 1(b)].

The probability density function $p(A)$ of the Voronoi cell areas has no known analytical expression, but can be well approximated by $p(A)=\frac{343}{15} \sqrt{7 / 2 \pi} A^{2.5} e^{-3.5 A}$, where $A$ denotes the normalized cell area [25]. This expression provides excellent fits to our arrays for all obstacle densities, and the average value $\langle A\rangle=1$ as well as the standard deviation $\sigma_{A} \approx \sqrt{2 / 7}$ are well reproduced [see Fig. 1(c)]. The obstacles in one array have identical shapes (circles, squares, and retroreflectors, see the insets in Fig. 2) and sizes characterized by a lateral extension $d_{s}=1$ and $2 \mu \mathrm{m}$, respectively. Here, $d_{s}$ is the diameter of the circles or the edge length of the squares and retroreflectors, respectively. For the implementation of an array, one shape is allocated with random orientation to each center coordinate by a pattern generator and then transferred to the samples by electron beam lithography and subsequent reactive ion etching. The etch depth of roughly $150 \mathrm{~nm}$ in all samples ensures depletion of the electron gas in the etched regions. An example of such an array is shown in Fig. 1(b).

From Aharonov-Bohm experiments on comparable structures in large magnetic fields [26], we estimate the lateral depletion length around the etched structure to $\approx 75 \mathrm{~nm}$. Taking this depletion into account, the corresponding densities of the arrays of circles and retroreflectors are $n^{\star}=0.065,0.13,0.195$, and 0.26 , while for the arrays of squares, the densities are smaller by a factor of $\pi / 4$. This choice of parameters ensures that on the one hand, the obstacle size is large compared to the Fermi wavelength of $\approx 50 \mathrm{~nm}$ such that the scattering can be regarded as classical, while on the other hand, the resistivity 


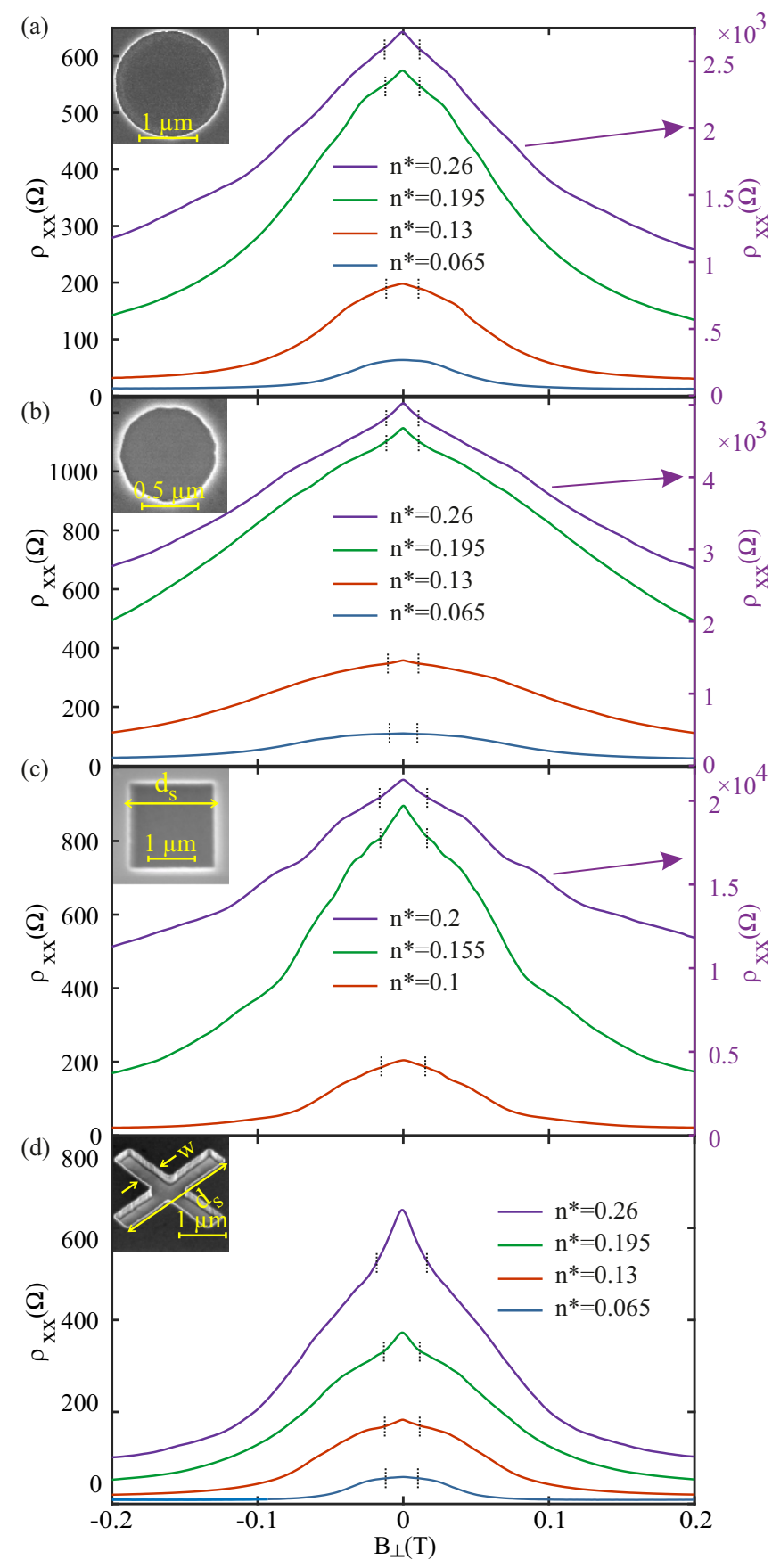

FIG. 2. Longitudinal magnetoresistance of the 2DLGs with various obstacle densities $n^{\star}$ for circles with $d_{s}=2 \mu \mathrm{m}$ (a) and $1 \mu \mathrm{m}$ (b), squares with $d_{s}=2 \mu \mathrm{m}$ (c), and retroreflectors with $d_{s}=2 \mu \mathrm{m}$ (d). The interval of the linear regime is indicated by the dashed, vertical lines. Scanning electron electron microscope pictures of the corresponding obstacles that form the arrays are shown in the insets. The temperature was $1.4 \mathrm{~K}$ in all measurements.

of the arrays is dominated by the patterned obstacles. The mean-free path of the Lorentz gas composed of circular disks in the dilute limit is given by $\ell_{s}=\left(n_{s} d_{s}\right)^{-1}$, corresponding to values below $8 \mu \mathrm{m}$ for all obstacle densities, significantly smaller than $\ell$. Only the arrays with the smallest $n_{s}$ values of $n_{s}=6.5 \times 10^{10} \mathrm{~m}^{-2}$ can be considered as dilute, which defines the range of validity of the models mentioned above, i.e., in Refs. [14-19]. Furthermore, the absolute number of obstacles varies between 975 and 20000 which should ensure reasonable ensemble averaging. All arrays are located in Hall bar structures and have a size of $100 \mu \mathrm{m} \times 200 \mu \mathrm{m}$. The insets in Fig. 2 illustrate the geometries of the individual obstacles.

Measurements are carried out in the mixing chamber of a ${ }^{3} \mathrm{He} /{ }^{4} \mathrm{He}$ dilution refrigerator with a base temperature of $25 \mathrm{mK}$ and in a ${ }^{4} \mathrm{He}$ gas flow cryostat that can be operated at temperatures between 1.4 and $300 \mathrm{~K}$. Both cryostats are equipped with superconducting solenoids and a rotatable sample stage. They are used for applying perpendicular magnetic fields $B_{\perp}$ as well as for in-plane components $B_{\|}$. An ac current (amplitude $500 \mathrm{nA}, 17.7 \mathrm{~Hz}$ ) is applied, and the magnetoresistance components $R_{x x}\left(B_{\perp}\right)$ and $R_{x y}\left(B_{\perp}\right)$ are measured via voltage probes using lock-in amplifiers.

\section{EXPERIMENTAL RESULTS AND DISCUSSION}

Figure 2 shows the measured longitudinal magnetoresistivities $\rho_{x x}\left(B_{\perp}\right)$ of the 2DLGs formed by circles $\left(d_{s}=1\right.$ and $2 \mu \mathrm{m})$ as well as by squares and retroreflectors with $d_{s}=$ $2 \mu \mathrm{m}$. A pronounced peak centered at $B_{\perp}=0$ is observed in all samples which, for larger $n^{\star}$, extends well into the regime where Shubnikov-de Haas oscillations are visible. For small $B_{\perp}$, the magnetoresistivity within the peak is approximately linear and develops a nonlinear behavior as $B_{\perp}$ increases. Note that the resistivity of the arrays of squares with the largest $n^{\star}$ is significantly larger than that one of the circles at the same obstacle density. We attribute this to the fact that the array of squares is much closer to the percolation threshold of $n_{c}^{\star}=0.246$ for the squares vs 0.359 for the circles [27] (the percolation threshold for the arrays of retroreflectors has not been determined yet to the best of our knowledge). This may also explain the structures visible in the flanks of the peaks in these samples since the proximity to the percolation threshold makes the transport more sensitive to local details of the potential.

At first sight, the magnetoresistance peaks resemble those observed on pristine high-mobility 2 DEGs, which are usually referred to as giant negative magnetoresistance (GNMR) [7-11]. This structure, which is also present in our pristine samples, shows, aside from a marked dependence on the width of the Hall bar [28], the parametric dependencies as reported earlier: a rapid suppression as the temperature is increased to $1 \mathrm{~K}$ as well as by parallel magnetic fields $[9,29]$. The amplitude of the GNMR structure in our samples is in the range of a few $\Omega$ only and thus negligible in the arrays containing artificial obstacles at all our obstacle number densities. Our data even suggest that already at our lowest obstacle density, the GNMR is strongly suppressed [29]. Furthermore, the dominant magnetoresistance peak observed in our samples does not depend significantly on in-plane $B$ fields and is much more robust with respect to thermal smearing.

As $n^{\star}$ is increased, the linear part of the peak tends to get more pronounced and increases both in amplitude and width. For large obstacle densities, the amplitude of the corresponding conductance dip is in the range of $2 e^{2} / h$, the maximum possible amplitude of weak localization. For low densities, however, the amplitude is significantly larger, reaching values 

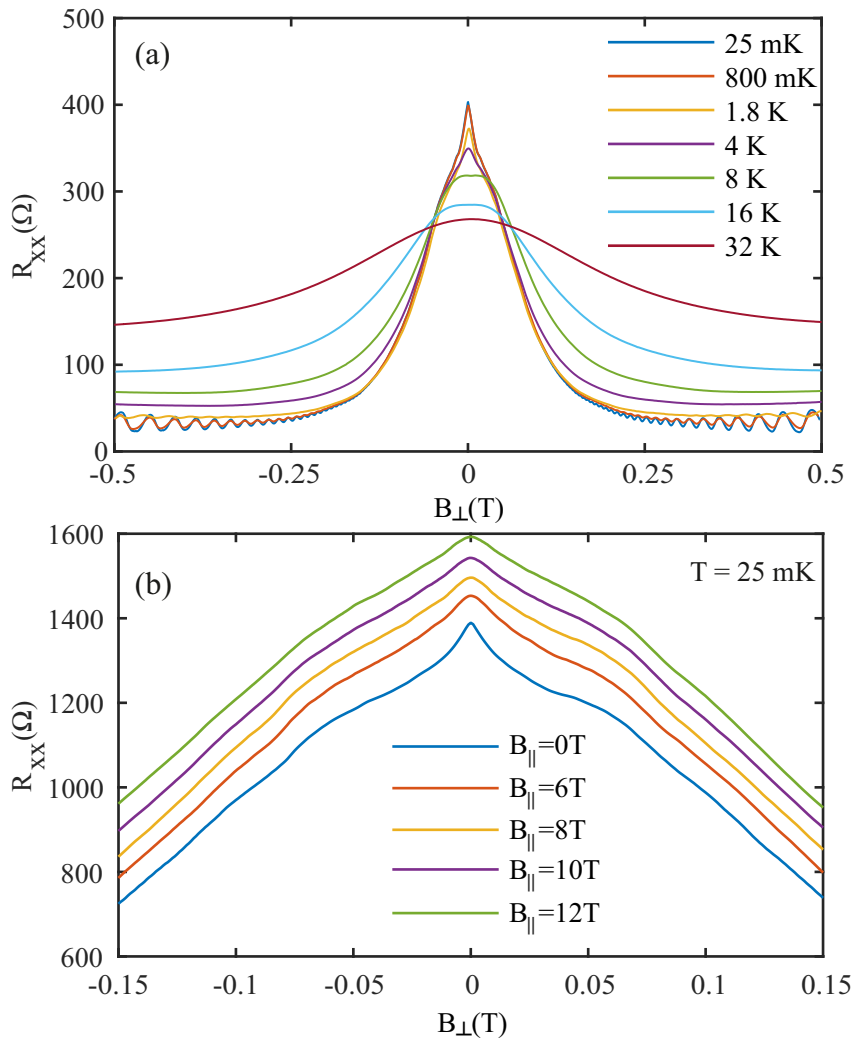

FIG. 3. (a) Temperature dependence of $R_{x x}$ for the array formed by retroreflectors with a density of $n^{\star}=0.195$ and $d_{s}=2 \mu \mathrm{m}$. (b) Effect of in-plane magnetic fields on the magnetoresistivity, for the array of circles with $d_{s}=1 \mu \mathrm{m}$ and $n^{\star}=0.195$. The traces are offset by $50 \Omega$ each for clarity.

as high as $\approx 50 e^{2} / h$ [29], which excludes weak localization as the dominant origin. Furthermore, the peak is more prominent in the Lorentz gases formed by retroreflectors in comparison to those formed by circles and squares of size $2 \mu \mathrm{m}$. We have also measured arrays of squares and retroreflectors with $d_{s}=1 \mu \mathrm{m}$ and found no significant differences to the corresponding arrays of circles [29]. We take this as an indication that for the obstacles with the smaller size, the geometric smearing of the obstacle shape by the lateral depletion is already significant and leads to a convergence of the scattering cross sections. In Fig. 3(a), the temperature dependence of the peak is exemplified using the retroreflector array of density $n^{\star}=0.195$. A qualitatively similar behavior is observed for the other arrays. As the sample is warmed from 25 to $800 \mathrm{mK}$, the peak shape remains nearly unaffected while the Shubnikov-de Haas oscillations show thermal smearing. This can be seen as an indication that weak localization does not dominate the peak shape. As the temperature is increased to $8 \mathrm{~K}$, the linear part of the peak, visible for magnetic fields below $30 \mathrm{mT}$ in this case, decays to zero amplitude. The background peak has a much weaker temperature dependence and is still clearly visible at $32 \mathrm{~K}$.

The influence of in-plane magnetic fields $B_{\|}$is shown in Fig. 3(b), exemplified for the array of circles with with $d_{s}=1 \mu \mathrm{m}$ and $n^{\star}=0.195$. These data have been obtained by rotating the samples with respect to the fixed magnetic field

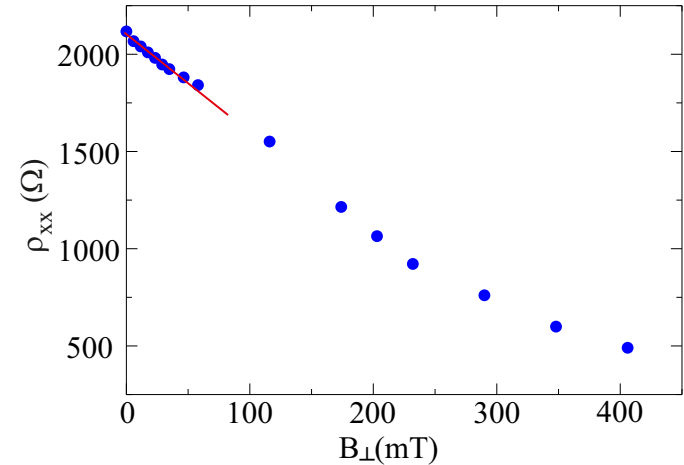

FIG. 4. Result of a model simulation (blue circles) of $\rho_{x x}(B)$ for a 2DLG formed by an array of circles $\left(d_{s}=1 \mu \mathrm{m}\right.$ and $\left.n^{\star}=0.225\right)$. The red line shows a linear approximation of the data points at the seven lowest magnetic fields $(B \leqslant 34 \mathrm{mT})$.

by angles up to $\approx 1.4^{\circ}$ around $\pi / 2$, such that a change of the perpendicular component $B_{\perp}$ by $\pm 0.15 \mathrm{~T}$ was reached under approximately constant $B_{\|}$(changes of less than $2 \mathrm{mT}$ ). The peak is suppressed as $B_{\|}$is increased and remains barely visible at $B_{\|}=12 \mathrm{~T}$.

In order to narrow down the character of this peak further, we have carried out molecular dynamics simulations for an array formed by circles with $d_{s}=1 \mu \mathrm{m}$ and $n^{\star}=0.225$. The electrons and scatterers interact via a standard shifted, purely repulsive Weeks-Chandler-Andersen (WCA) potential, $u(r)=4 \varepsilon\left[\left(r_{s} / r\right)^{12}-\left(r_{s} / r\right)^{6}+1 / 4\right]$ for $r<2^{1 / 6} r_{s}$ and $u(r)=0$ otherwise. Here, we have set the energy parameter to $\varepsilon=0.1 E_{F}$. We use 100 statistically independent matrix structures. Newton's equations of motion are integrated using the velocity-Verlet algorithm [30] with a time step of $10^{-3} t_{0}$ with $t_{0}$ being the time an electron needs to travel ballistically the distance $d_{s}$, i.e., $t_{0}=9.23$ ps for our samples. All electrons move with the Fermi velocity in regions where $u=0$. At each magnetic field, 2400 electrons are used for runs of $10^{6} t_{0}$ duration. In Fig. 4, the result of such a model calculation is shown. Both features of the magnetoresistance, namely, the broad shoulder and the smaller, approximately linear, peak close to $B_{\perp}=0$, are reproduced. The linear regime extends up to $B_{\perp} \approx 30 \mathrm{mT}$, which is of the same order of magnitude as observed experimentally for comparable parameters. A linear negative magnetoresistivity is also obtained in corresponding simulations for lower $n^{\star}$ (not shown). These model calculations demonstrate that classical effects can generate the measured structure also in the regime of large obstacle densities and justifies its discussion in terms of memory effects.

We attribute the broad background component of the peak predominantly to undisturbed cyclotron motion of electrons in-between the obstacles [15]. This structure is of no further interest here. Rather, we focus on the approximately linear peak close to $B_{\perp}=0$. As mentioned in the Introduction, theoretical studies were carried out for low obstacle densities [18], showing that retroreflection causes the resistance to decrease linearly as a function of $B_{\perp}$ in the regime $\omega_{c} \tau_{s} \lesssim 0.2$ where $\omega_{c}$ denotes the cyclotron frequency and $\tau_{s}$ the obstaclegenerated scattering time, i.e., $\tau_{s}=\left(n_{s} d_{s} v_{F}\right)^{-1}$ with the Fermi velocity $v_{F}$. Here, $d_{s}$ takes the role of the total scattering 
cross section for circles. These results have been derived for the limit of low obstacle densities, characterized by $d_{s} \ll \ell_{s}$ with the obstacle-dominated mean-free path of $\ell_{s}=v_{F} \tau_{s}$. Interestingly, the normalized slope $\alpha$ of $\rho_{x x}(\perp)$, defined as

$$
\alpha \equiv \frac{1}{\rho_{x x}(0) \tau_{s}} \frac{d\left[\delta \rho_{x x}\left(B_{\perp}\right)\right]}{d \omega_{c}}
$$

is independent of the obstacle density in this regime and has a value which is characteristic for the obstacle shape. For circular obstacles, $\alpha_{\circ}=-0.04$ has been reported [18], while to the best of our knowledge, other shapes have not yet been considered in this context. The numerical studies have shown that such a linear magnetoresistivity is still to be expected at relatively large obstacle densities of $n^{\star} \approx 0.05$. These results are in agreement with the experiments of Gusev et al. in Ref. [23]. The fact that the obstacle positions were not random but showed Gaussian deviations from a periodic arrangement is of minor relevance in that context since, at low obstacle densities, retroreflection is a single-obstacle process.

In Fig. 5(a), we show the normalized magnetoresistivities for the arrays presented in Fig. 2. Here, we have followed Ref. [18] and determined the scattering times according to $\tau_{s}=\left(n_{s} \sigma_{s} v_{F}\right)^{-1}$, where $\sigma_{s}$ is the total scattering cross section of one obstacle, i.e., $\sigma_{s, \circ}=d_{s}, \sigma_{s, \square}=\frac{4 d_{s}}{\pi}$, and $\sigma_{s, \times}=$ $\frac{2}{\pi}\left[(2-\sqrt{2}) w+\sqrt{2} d_{s}\right] \approx 0.98 d_{s}$, where $w$ denotes the width of the retroreflector bars (see also Fig. 2), in our case $w=$ $0.45 \mu \mathrm{m}$ for the samples with $d_{s}=2 \mu \mathrm{m}$ and $w=0.3 \mu \mathrm{m}$ for the samples with $d_{s}=1 \mu \mathrm{m}$, respectively. A linear regime is found for all arrays, with an interval of varying size, extending up to $\omega_{c} \tau_{s} \approx 0.4,0.5$, and 0.9 for the arrays of retroreflectors, squares, and circle with $d_{s}=2 \mu \mathrm{m}$, respectively. The array formed by circles with $d_{s}=1 \mu \mathrm{m}$ shows a smaller interval, with an upper limit of $\omega_{c} \tau_{s} \approx 0.2$. This is in rough agreement with the interval sizes of $\omega_{c} \tau_{s} \approx 0.2$ found in numerical simulations for the dilute case $[18,19]$, as well as with the results of our molecular dynamics simulations (see Fig. 4). Within the retroreflection picture, it corresponds to the magnetic field where the overlap between the ballistic corridors of injected and retroreflected electrons becomes insignificant.

The values of $\alpha$ are plotted as a function of the scattering times in Fig. 5(b). Here, $\tau_{0}$ denotes the time the electron needs to move ballistically the distance $d_{s}$, i.e., $\tau_{0}=4.3 \mathrm{ps}$ for $d_{s}=$ $1 \mu \mathrm{m}$ and $\tau_{0}=8.6 \mathrm{ps}$ for $d_{s}=2 \mu \mathrm{m}$. As $\tau_{s} / \tau_{0}$ reaches values of 2 or above, $\alpha$ is comparable to the value of 0.04 as predicted theoretically for the dilute case [18]. For all obstacle shapes, $\alpha$ increases as $\tau_{s}$ is decreased. This increase is largest for the retroreflectors with $d_{s}=2 \mu \mathrm{m}$, reaching a value as high as $\alpha_{\times}=0.48$ for the sample with $n^{\star}=0.26$.

These observations are interesting in two respects. First of all, they represent an experimental verification of the suggestion by theory that $\alpha$ is a measure of the retroreflectivity of the individual obstacle. While our data do not show a clear difference in this respect between circles and squares, $\alpha$ is enhanced for the retroreflectors with $d_{s}=2 \mu \mathrm{m}$ in comparison to the arrays of circles and squares of the same size. Second, if $\alpha$ is interpreted this way, our data suggest that the retroreflectivity increases with increasing obstacle density. We are not aware of any theoretical work in relation to this issue, but provide a simple geometrical interpretation, illustrated in the inset of
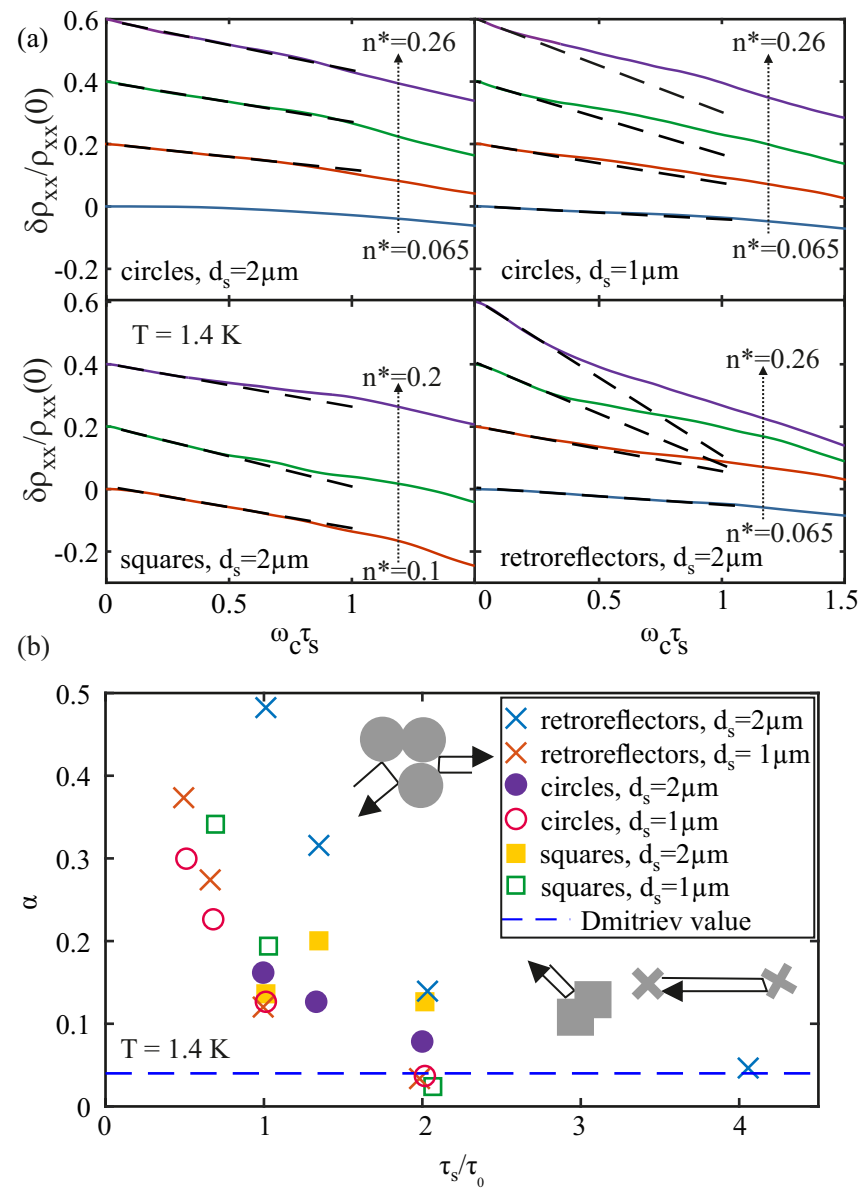

FIG. 5. (a) Experimentally determined normalized magnetoresistivity of the studied arrays and the corresponding linear fits for the different arrays. The traces at larger $n^{\star}$ are vertically offset by 0.2 for clarity. (b) Normalized slopes $\alpha$ of the normalized linear negative magnetoresistivities as a function of $n^{\star}$. The inset shows exemplified retroreflected trajectores at clusters of circles and squares, as well as a bound trajectory in a resonator formed by two retroreflectors. All data have been obtained for $T=1.4 \mathrm{~K}$.

Fig. 5(b). As $n^{\star}$ increases, the probability of finding two obstacles close together or even overlapping increases as well. This leads to additional backscattering (in the sense of Ref. [18]) when the corridor of the incoming electron overlaps with one of an outgoing electron after multiple reflections at more than one obstacle, i.e., for spatial separations of the two trajectories smaller than $d_{s}$. The contribution of this effect could be tested by future studies on arrays with increased obstacle density but with a different spatial distribution statistics, for example, by excluding overlaps or by periodic arrays with large Gaussian disorder. In addition, in particular for the retroreflector arrays, the formation of random resonators that localize electrons in-between two obstacles will increase as the scatterer density increases. Electrons may scatter into or out of such resonators by random background scattering on phonons or residual impurities. A clarification of how these effects contribute quantitatively to $\alpha$ requires extensive theoretical studies that are beyond the scope of this work.

In Fig. 6, the dependencies of $\alpha$ on the temperature and on in-plane magnetic fields $B_{\|}$are shown. We observe that, 


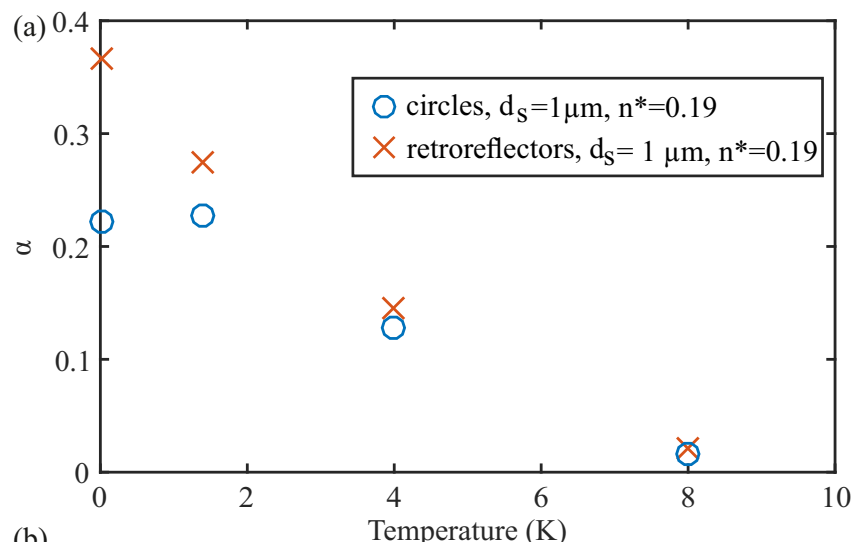

(b)

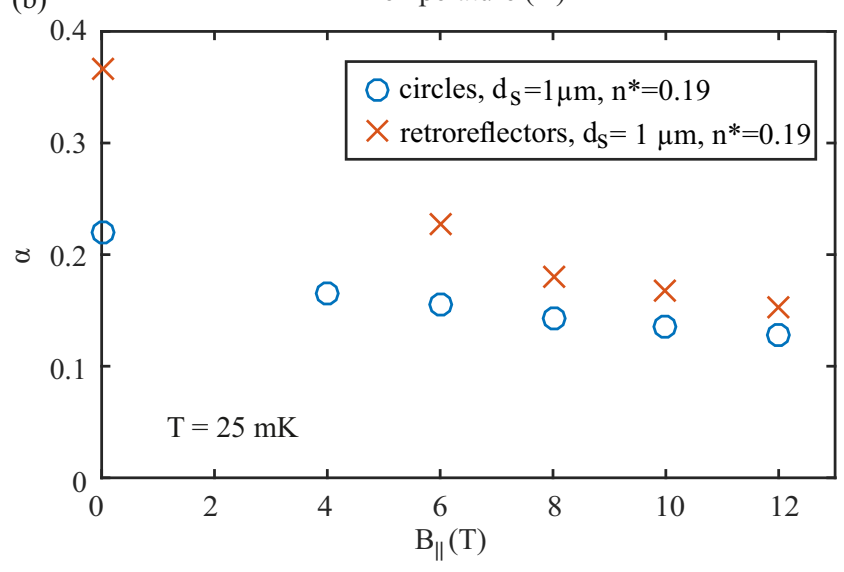

FIG. 6. Dependence of $\alpha$ on the temperature (a) and on in-plane magnetic fields $B_{\|}(\mathrm{b})$, exemplified by two arrays (see Fig. 3 for examples of the raw data).

in general, $\alpha$ decays as $T$ is increased and vanishes at a temperature of about $8 \mathrm{~K}$. Among other effects like thermal smearing, this may originate from acoustic phonons that scatter the electrons off the ballistic corridors, thereby decreasing their length. This is in tune with our observation that the mean-free path $\ell$ decreases from $31 \mu \mathrm{m}$ at $25 \mathrm{mK}$ to $20 \mu \mathrm{m}$ at $8 \mathrm{~K}$, the largest temperature where the peak can still be analyzed. Yevtushenko et al. [3] have reported a much weaker temperature dependence of the corresponding peak in their arrays, which remained visible up to temperatures of $44 \mathrm{~K}$. This may be due to the significantly smaller obstacle sizes of $d_{s} \approx 200 \mathrm{~nm}$ in those arrays, as well as due to the possible domination of these peaks by ballistic weak localization. Furthermore, in Ref. [3], the conductivity amplitude of this peak is studied as a function of temperature, which leads to its interpretation in terms of ballistic weak localization with the Ehrenfest and the dephasing times as characteristic time scales [31]. In our data, such an analysis is hampered by the gradual and temperature-dependent transition between the linear and the background peaks, which impedes a meaningful subtraction of some background conductance.

A decrease of $\alpha$ is also observed as a function of increasing $B_{\|}$[see Fig. 6(b)]. For the retroreflectors, a rapid suppression of the peak is observed as $B_{\|}$is increased from zero to $\approx 8 \mathrm{~T}$, while at a further increase of $B_{\|}$, the suppression is less pronounced. For the circles, the suppression of the peak by $B_{\|}$is weaker. Also, the $\alpha$ values of different obstacle shapes tend to converge towards similar values at large in-plane magnetic fields. In relation to the well-known suppression of the conventional weak localization peak [2], it has been established that due to the diamagnetic shift, the increase of the electron density of states and the modification of electron wave functions, the electrons develop a larger sensitivity to the roughness of the GaAs- $\mathrm{Al}_{\mathrm{x}} \mathrm{Ga}_{1-\mathrm{x}} \mathrm{As}$ interface which causes a reduction of the mean-free path [32]. Also, $B_{\|}$is known to deform the cyclotron orbits in a characteristic way [33]. In the pristine 2DEG, we observe a decrease of $\ell$ from $31 \mu \mathrm{m}$ at $B_{\|}=0$ to $16 \mu \mathrm{m}$ at $B_{\|}=12 \mathrm{~T}$, which demonstrates that in fact a process is active which suppresses the mobility in parallel magnetic fields. This effect leads to a modified overlap of the incoming and reflected corridors. A model for the effect of in-plane magnetic fields on retroreflection is presently not available. However, it appears plausible that the additional scattering generated by $B_{\|}$also reduces the extension of the ballistic corridors, thereby suppressing the retroreflection. The observed convergence of the $\alpha$ values for different obstacle shapes as $B_{\|}$is increased is in tune with this picture, considering that $B_{\|}$ effectively shortens the ballistic corridor and thus reduces the relevance of the obstacle-specific reflectivity.

All results presented above are influenced not only by the artificial array of obstacles, but also by the background disorder. Effects of background disorder on memory effects have been discussed so far, to the best of our knowledge, only in relation to the depinning of electrons from single obstacles or obstacle clusters [34]. The small-angle (quantum) scattering time of $\tau_{q}$ implies that the electrons undergo a random scattering event after traveling an average distance of $\ell_{q}=530 \mathrm{~nm}$ in our samples. These small deflections can scatter the electrons out of the retroreflection corridor, thereby limiting its length. Thus, small-angle scattering may contribute to our observation that retroreflection is more visible in arrays with larger obstacle density where the ballistic corridors are shorter. A quantitative description of these effects is beyond the scope of this paper and requires extensive numerical simulations. However, since we expect that quite a few small-angle deflections will be necessary to remove the electron from the reflective corridor, the electrons will still probe a significant fraction of the overlap of the corridors for the incoming and the reflected electrons, namely, over a length scale comparable to the distance between the artificial obstacles.

It should be noted that a parabolic magnetoresistivity has been predicted for very small magnetic fields as a consequence of memory effects [19]. For our samples, the upper magnetic field limit for this interval is of the order of $100 \mu \mathrm{T}$ and thus not resolvable in the measurements reported here. Furthermore, for $\omega_{c} \tau_{s} \ll n_{s} d_{s}^{2}$, a $B_{\perp}^{-1 / 2}$ dependence has been predicted [19]. We do not find such a dependence in any of our samples. This could be due to the relatively large values of $n^{\star}$ in our samples, or a consequence of distortions by residual background scattering [34].

We end this section by returning to the GNMR effect and its relation to our measurements. Typical GNMR data show a broad peak in $\rho_{x x}\left(B_{\perp}\right)$ with the phenomenology as described above, plus a small peak close to $B_{\perp}=0$ on top, which has been attributed to sparse oval defects that act as Lorentz array and shows a remarkable robustness with respect to elevated temperatures [8] as well as to in-plane magnetic fields $[8,29]$. 
Within this picture, it would thus correspond to the broad maximum we observe in our 2DLGs. On the other hand, the small peak we interpreted in terms of retroreflection is not resolved in the GNMR measurements.

\section{SUMMARY AND CONCLUSIONS}

A linear negative magnetoresistance is observed in twodimensional Lorentz gases in the classical regime, in qualitative agreement with both the predicted magnetoresistivity due to retroreflection at the obstacles at small scatterer densities and a numerically calculated classical magnetoresistivity at large scatterer densities. It can be concluded that retroreflection, one member of the family of classical memory effects, is the dominant origin of this peak in our samples. A comprehensive phenomenology of this peak has been presented, including its dependence on the density and shape of the obstacles, on the temperature as well as on in-plane magnetic fields. The measurements confirm the existing theory which has focused on circular-shaped obstacles in the low-density regime. However, the normalized slope $\alpha$ of the magnetoresistance decreases as the temperature or the in-plane magnetic field is increased and increases with a larger density or retroreflectivity of the obstacles. These results cannot be understood within the presently available models. We have tentatively explained the behavior of $\alpha$ as a function of the obstacle density in terms of additional retroreflection that emerges due to the formation of clusters and/or resonators by the obstacles. Although our molecular dynamics simulations suggest that retroreflection does contribute to the experimentally observed structure, we cannot exclude an additional contribution by ballistic weak localization, which, however, would be non-negligible only for arrays with large obstacle densities. A further clarification would require theoretical work that extends to larger obstacle densities and includes the effects of the obstacle shape as well as of in-plane magnetic fields.

\section{ACKNOWLEDGMENT}

This project has received funding from the EU-H2020 research and innovation programme under Grant Agreement No. 654360.
[1] K. von Klitzing, G. Dorda, and M. Pepper, Phys. Rev. Lett. 45, 494 (1980).

[2] B. L. Altshuler, A. G. Aronov, and D. E. Khmelnitsky, J. Phys. C: Solid State Phys. 15, 7367 (1982).

[3] O. Yevtushenko, G. Lütjering, D. Weiss, and K. Richter, Phys. Rev. Lett. 84, 542 (2000).

[4] I. L. Aleiner and A. I. Larkin, Phys. Rev. B 54, 14423 (1996).

[5] M. A. Paalanen, D. C. Tsui, and J. C. M. Hwang, Phys. Rev. Lett. 51, 2226 (1983).

[6] P. S. Alekseev, Phys. Rev. Lett. 117, 166601 (2016).

[7] V. Umansky, R. de Picciotto, and M. Heiblum, Appl. Phys. Lett. 71, 683 (1997).

[8] L. Bockhorn, P. Barthold, D. Schuh, W. Wegscheider, and R. J. Haug, Phys. Rev. B 83, 113301 (2011).

[9] A. T. Hatke, M. A. Zudov, J. L. Reno, L. N. Pfeiffer, and K. W. West, Phys. Rev. B 85, 081304(R) (2012).

[10] L. Bockhorn, I. V. Gornyi, D. Schuh, C. Reichl, W. Wegscheider, and R. J. Haug, Phys. Rev. B 90, 165434 (2014).

[11] Q. Shi, P. D. Martin, Q. A. Ebner, M. A. Zudov, L. N. Pfeiffer, and K. W. West, Phys. Rev. B 89, 201301(R) (2014).

[12] A. D. M. A. Dmitriev, D. G. Polyakov, and M. A. Zudov, Rev. Mod. Phys. 84, 1709 (2012).

[13] H. Lorentz, Proc. R. Acad. Sci. Amsterdam 7, 438 (1905).

[14] E. M. Baskin, L. N. Magarill, and M. V. Entin, Zh. Eksp. Teor. Fiz. 75, 723 (1978) [Sov. Phys.-JETP 48, 365 (1978)].

[15] A. V. Bobylev, F. A. Maaø, A. Hansen, and E. H. Hauge, Phys. Rev. Lett. 75, 197 (1995).

[16] A. D. Mirlin, D. G. Polyakov, F. Evers, and P. Wölfle, Phys. Rev. Lett. 87, 126805 (2001).

[17] D. G. Polyakov, F. Evers, A. D. Mirlin, and P. Wölfle, Phys. Rev. B 64, 205306 (2001).

[18] A. Dmitriev, M. Dyakonov, and R. Jullien, Phys. Rev. Lett. 89, 266804 (2002).
[19] V. V. Cheianov, A. P. Dmitriev, and V. Y. Kachorovskii, Phys. Rev. B 68, 201304 (R) (2003).

[20] A. Kuzmany and H. Spohn, Phys. Rev. E 57, 5544 (1998).

[21] W. Schirmacher, B. Fuchs, F. Höfling, and T. Franosch, Phys. Rev. Lett. 115, 240602 (2015).

[22] N. H. Siboni, J. Schluck, K. Pierz, H. W. Schumacher, D. Kazazis, J. Horbach, and T. Heinzel, Phys. Rev. Lett. 120, 056601 (2018).

[23] G. M. Gusev, P. Basmaji, Z. D. Kvon, L. V. Litvin, Y. Nastaushev, and A. I. Toropov, J. Phys.: Condens. Matter 6, 73 (1994).

[24] T. Ando, J. Phys. Soc. Jpn. 37, 1044 (1974).

[25] J. Ferenc and Z. Neda, Phys. A (Amsterdam) 385, 518 (2007).

[26] J. Schluck, S. Fasbender, T. Heinzel, K. Pierz, H. W. Schumacher, D. Kazazis, and U. Gennser, Phys. Rev. B 91, 195303 (2015).

[27] S. Mertens and C. Moore, Phys. Rev. E 86, 061109 (2012).

[28] R. G. Mani, A. Kriisa, and W. Wegscheider, Sci. Rep. 3, 02747 (2013).

[29] See Supplemental Material at http://link.aps.org/supplemental/ 10.1103/PhysRevB.97.115301 for additional raw data and information regarding the giant negative magnetoresistance in our samples.

[30] K. Binder, J. Horbach, W. Kob, W. Paul, and F. Varnik, J. Phys.: Condens. Matter 16, S429 (2004).

[31] A. Altland, P. W. Brouwer, and C. Tian, Phys. Rev. Lett. 99, 036804 (2007)

[32] G. M. Minkov, O. E. Rut, A. V. Germanenko, A. A. Sherstobitov, B. N. Zvonkov, V. I. Shashkin, O. I. Khrykin, and D. O. Filatov, Phys. Rev. B 70, 035304 (2004).

[33] N. M. Sotomayor-Choque, G. M. Gusev, J. R. Leite, A. A. Bykov, L. V. Litvin, N. T. Moshegov, A. I. Toropov, D. K. Maude, and J. C. Portal, Phys. Rev. B 66, 035324 (2002).

[34] A. D. Mirlin, E. Tsitsishvili, and P. Wölfle, Phys. Rev. B 63, 245310 (2001). 